\title{
Mathematical derivation
}

Our mathematical model is based on 1D fluid dynamic shear models (e.g. Turcotte and Schubert, 2002). We apply a constitutive equation in the form of a dislocation creep flow law

$$
\dot{\gamma}=A e^{\left(\frac{-Q}{R T}\right)} \sigma^{n}
$$

where $\dot{\gamma}=\partial v / \partial \mathrm{z}$ is the shear strain rate, $\mathrm{z}$ is the vertical coordinate across the shear zone being zero at the base and increasing positively upwards, $\mathrm{v}$ is the horizontal (i.e. parallel to the shear zone) velocity, $\sigma$ is the shear stress, $\mathrm{A}$ is a pre-exponential factor, $n$ is the power-law stress exponent, $\mathrm{Q}$ is the activation energy, $\mathrm{R}$ is the gas constant and $\mathrm{T}$ is the temperature. We solve (1) for $\sigma$ which yields

$$
\sigma=\left[A e^{\frac{-Q}{R T}}\right]^{-1 / n} \dot{\gamma}^{1 / n}
$$

This can be more generally expressed as

$$
\sigma=\mu \dot{\gamma}^{1 / n}
$$

where $\mu$ is a viscosity coefficient. We assume a normal geotherm and, hence, that the temperature increases linearly with depth inside the shear zone

$$
T=T_{0}-\theta z
$$

where $T_{0}$ is the temperature at the base of the shear zone and $\theta$ is the temperature gradient. Substituting (4) into (2) provides the viscosity factor in (3):

$$
\mu=A^{-1 / n} e^{\frac{Q}{n R\left(T_{0}-\theta z\right)}}
$$

It is difficult to derive an analytical solution for terms of the form $e^{1 / z}$ as in (5). A common simplification is to use the Frank-Kamenetzky approximation which employs a term of the form $e^{z}$. The alternative viscosity coefficient $\eta$ has then the form

$$
\eta=\eta_{0} e^{z / \lambda}
$$


where $\eta_{0}$ is the coefficient at the base of the shear zone $(\mathrm{z}=0)$ and $\lambda$ is the e-fold length. $\lambda$ quantifies the distance over which the value of $\eta$ increases by a factor of e. The material parameters in (5) derived from laboratory rock deformation experiments can be related to the new parameters $\eta_{0}$ and $\lambda$. The deformation is most intense at the base of the shear zone and, therefore, we want that $\mu$ is well approximated by $\eta$ at the base. We require that both the values and the spatial derivatives of $\mu$ and $\eta$ are identical at $\mathrm{z}=0$ which provides two equations:

$$
\begin{aligned}
& \mu_{z=0}=\eta_{z=0} \\
& {\left[\frac{\partial \mu}{\partial z}\right]_{z=0}=\left[\frac{\partial \eta}{\partial z}\right]_{z=0}}
\end{aligned}
$$

The explicit form of these equations is

$$
\begin{aligned}
& A^{-1 / n} e^{\frac{Q}{n R T_{0}}}=\eta_{0} \\
& \frac{A^{-1 / n} Q \theta e^{\frac{Q}{n R T_{0}}}}{n R T_{0}^{2}}=\frac{\eta_{0}}{\lambda}
\end{aligned}
$$

Solving the two equations yields

$$
\begin{aligned}
& \eta_{0}=A^{-1 / n} e^{\frac{Q}{n R T_{0}}} \\
& \lambda=\frac{n R T_{0}^{2}}{Q \theta}
\end{aligned}
$$

The expression for the stress can now be written as

$$
\sigma=\eta_{0} e^{\frac{z}{\lambda}}\left(\frac{\partial v}{\partial z}\right)^{1 / n}
$$

We consider a 1D horizontal shear zone where the vertical velocities are zero. We assume that horizontal pressure gradients are negligible and, therefore, the 1D force balance is (e.g. Turcotte and Schubert, 2002)

$$
\frac{\partial \sigma}{\partial z}=0
$$


Substituting (10) into (11) and using the two kinematic boundary conditions $v_{z=0}=v_{0}$ and $v_{z=W}=0$, with $\mathrm{W}$ being the shear zone width, yields the velocity

$$
v=\frac{v_{0}\left(e^{\frac{n(W-z)}{\lambda}}-1\right)}{e^{\frac{n W}{\lambda}}-1}
$$

We make the solution for $\mathrm{v}$ dimensionless by introducing

$$
\begin{aligned}
& \beta=\frac{n W}{\lambda}=\frac{Q \theta W}{R T_{0}^{2}} \\
& \bar{v}=v / v_{0} \\
& \bar{Z}=\mathrm{z} / W
\end{aligned}
$$

$\bar{Z}$ is the non-dimensional vertical coordinate, and $\bar{v}$ is the non-dimensional horizontal velocity. The value of $\beta$ depends on the thickness of the shear zone, W. Using $\theta=\Delta T / W$ with $\Delta \mathrm{T}$ being the temperature difference between the temperature at the bottom and the top of the shear zone provides an expression for $\beta$ independent on $\mathrm{W}$ :

$$
\beta=\frac{Q}{R T_{0}} \frac{\Delta T}{T_{0}}
$$

The value of $\beta$ does not depend on the material properties $n$ and $\eta_{0}$ but only on the parameters Q, $\Delta T$ and $T_{0}$. The dimensionless velocity now depends only on $\beta$ :

$$
\bar{v}=\frac{e^{\beta(1-\bar{z})}-1}{e^{\beta}-1}
$$

The larger the value of $\beta$ the stronger the strain localizes (Fig. 2). 